\section{Úber die Bromierung von Acetanilid mit Brommonochlorid. Anwendung zur Bestimmung von Acetanilid}

Bromination of Acetanilide by Bromine Monochloride. Application for the Determination of Acetanilide

\section{P. KeRTÉsz und K. FörHẃCz}

Institut für Pharmazeutische Chemie, Semmelweis Medizinische Universität, Budapest, Ungarn

Eingegangen am 13. Oktober 1971

Durch Bromierung mit Brommonochlorid ist es uns gelungen, ein neues Verfahren zur quantitativen Bestimmung von Acetanilid auszuarbeiten. Die Identifizierung des Reaktionsproduktes lieferte indirekt - eine neue experimentelle Angabe für die Konformationsverhältnisse von Acetanilid.

\section{Quantitative Bestimmung}

Acetanilid reagiert unter den unten angegebenen Bedingungen unmittelbar quantitativ mit Brommonochlorid. Brommonochlorid entsteht aus einer $0,1 \mathrm{~N}$,,neutralen Brommonochloridlösung" auf Einwirkung von Salzsäure [1]. Die Bromierung ist bei einer Salzsäurekonzentration $1-2 \mathrm{~N}$ in 5 min fast vollständig. In weiteren $30 \mathrm{~min}$ verändern sich die Ergebnisse nicht. Obwohl die Menge der Meßlösung auch in breiten Grenzen (50-200\%)ohne Einfluß ist, empfehlen wir die Anwendung eines etwa 100\% igen Überschusses. Tageslicht beeinflußt die Ergebnisse nicht.

Arbeitsweise. Etwa 30-40 mg Substanz werden in einem $100 \mathrm{ml}-K o l b e n$ in 1-2 $\mathrm{ml}$ Eisessig gelöst, dann die Lösung mit $15 \mathrm{ml}$ Wasser verdünnt. Nach Zugabe von $20,00 \mathrm{ml}$ $0,1 \mathrm{~N}$, neutraler Brommonochloridlösung" werden $10 \mathrm{ml}$ $20 \%$ ige Salzsäure hinzugefügt. Nach 10 min wird im Reaktionsgemisch 0,5 g Kaliumjodid gelöst und das Jod nach Zusatz von Stärkelösung mit $0,1 \mathrm{~N}$ Natriumthiosulfatlösung titriert. $1 \mathrm{ml} \mathrm{0,1} \mathrm{N}$,neutrale Brommonochloridlösung“ entspricht $3,379 \mathrm{mg}$ Acetanilid. Der relative Fehler beträgt: $\pm 0,5 \%$.

$0,1 \mathrm{~N}$,neutrale Brommonochloridlösung". 2,7835 g Kaliumbromat und $3,9670 \mathrm{~g}$ Kaliumbromid mit Wasser auf $1000 \mathrm{ml}$ auffüllen.

\section{Untersuchung des Reaktionsproduktes}

Der bereits bei der Bestimmung beobachtete Bromverbrauch (Äquivalenzgewicht $=$ Molgewicht/4) läßt eine Dibromsubstitution annehmen.
Der Bromgehalt des während der Bestimmung ausgeschiedenen weißen Niederschlages - bestimmt nach Verbrennung mit der Bromcyanmethode [2] — betrug 53,91\%. (Theoretischer Bromgehalt des Dibromacetanilids: $\mathbf{5 4 , 5 5 \%} \%$.)

Der Schmelzpunkt des Produktes $\left(144,3^{\circ} \mathrm{C}\right)$ stimmt mit dem in der Literatur angegebenen Schmelzpunkt von 2,4-Dibromacetanilid überein. Das Hydrolyseprodukt dieses Dibromderivats erwies sich als 2,4-Dibromanilin (Schmelzpunkt: $79,5^{\circ} \mathrm{C}$ ). Dies kann durch bromometrische Bestimmung quantitativ in $2,4,6$-Tribromanilin (Schmelzpunkt: $118,8^{\circ} \mathrm{C}$ ) umgewandelt werden.

\section{Diskussion}

Nach den in der Literatur angegebenen Daten führt die Bromierung von Acetanilid zu einem Gemisch von bromierten Derivaten, oder zu p-Bromacetanilid.Nun konnte bewiesen werden, daß aus Acetanilid auf Einwirkung von Brommonochlorid unter den oben angegebenen Umständen quantitativ 2,4-Dibromacetanilid entsteht. Die Konformationsverhältnisse im Acetanilid und in seinen Derivaten wurden nach verschiedenen Gesichtspunkten untersucht. Demnach ist im Acetanilid selbst und in seinen an $\mathrm{C}_{2}$ substituierten Derivaten bezüglich der $\mathrm{C}_{1}-\mathrm{N}-\mathrm{C}=\mathrm{O}$ Atomgruppe die endo-Konformation am günstigsten, das heißt die Konformation, wo sich $\mathrm{C}_{1}$ und $\mathrm{O}$ in cisKonstellation befinden. Es ist anzunehmen, daß im 2,4-Dibromacetanilid die Rotation der planaren Acetamidogruppe um die Achse $\mathrm{C}_{1}-\mathrm{N}$ infolge der sterischen Wechselwirkung zwischen $\left(\mathrm{C}_{2}\right)-\mathrm{Br}$ und (N) - H sehr beschränkt ist. Die dadurch entstandene noch engere Nachbarschaft des Sauerstoffs schirmt das $\left(\mathrm{C}_{6}\right)-\mathrm{H}$ gegen den Angriff des Bromkations ab. In diesem Sinne kann die asymmetrische Bromsubstitution befriedigend erklärt werden.

Wir danken Herrn Prof. Dr. A. Végh, dem Direktor des Instituts, für die Förderung dieser Arbeit.

\section{Literatur}

1. Schulek, E., Burger, K.: Talanta 1, 219 (1958); vgl. diese Z. 169, 378 (1959).

2. - Ladányi, L.: Anal. Chim. Acta 27, 40 (1962); vgl. diese Z. 197, 385 (1963).

\section{P. Kertész}

Institut für Pharmazeutische Chemie Semmelweis Medizinische Universität Budapest, VIII.

Puskinstr. 11-13, Ungarn 